\title{
Importancia del currículo, texto y docente en la clase de matemática
}

Fecha de recepción : 31 de marzo de 2020 • Fecha de aceptación: 22 de abril de 2020 • Fecha de publicación: 10 de mayo de 2020

Fabián Eugenio Bravo Guerrero'

Universidad de Cuenca

fabianbravo@yahoo.com

https://orcid.org/0000-0002-0372-2071

\section{Resumen}

El ajuste curricular realizado en el Ecuador en 2016 responde al paradigma constructivista, los textos que guían al docente de matemática propuestos por el Ministerio de Educación también están desarrollados acorde a esa visión, entonces, el docente debe desarrollar una clase activa y reflexiva, apoyado en una serie de recursos propuestos en el ajuste curricular. La educación debe tener todos sus elementos bien armonizados para que el estudiante tenga los resultados de aprendizaje adecuados, se plantea el objetivo de analizar la relación entre tres elementos: diseño curricular, texto guía y el docente. En esta investigación se aplica la metodología de revisión documental para analizar cómo el ajuste curricular idealiza la relación entre estos elementos, y cuál es la realidad. En la práctica se encuentran algunas dificultades, una de ellas, los textos, que traen cantidades excesivas de contenidos a desarrollarse y obligan al docente a "dar" clases, sin cerciorarse del logro de aprendizajes de los alumnos; también, la reforma no ha tenido la suficiente difusión a los docentes, quienes aún desarrollan sus clases de forma tradicional, enfatizando en la memorización de contenidos y de recetas mecánicas para resolver los ejercicios. 


\begin{abstract}
The curricular adjustment carried out in Ecuador in 2016 responds to the constructivist paradigm, the texts that guide the mathematics teacher proposed by the Ministry of Education are also developed according to that vision, and therefore, the teacher should develop an active and reflective class, supported by a series of resources proposed in the curricular adjustment. Education must have all its elements well harmonized so that the student has the appropriate learning results; the objective is to analyze the relationship between three elements: curriculum design, guide text and the teacher. The documentary review methodology is applied to analyze how the curricular adjustment idealizes the relationship between these elements, and then what is the reality. In practice there are some difficulties, one of these is the texts bring excessive amounts of content to be developed, which forces the teacher to "give" classes, without making sure of the students' learning achievement. Also, the reform has not been sufficiently disseminated to teachers, who still develop their classes in a traditional way, emphasizing the memorization of content and mechanical recipes to solve the exercises.
\end{abstract}

Keywords: constructivism, mathematics teacher, education, 2016 curriculum reform, guide text. 


\section{Introducción}

En Ecuador se realizaron varias reformas a sus planes curriculares debido a que los estudiantes no lograban adquirir los saberes y habilidades que se proponía el Ministerio de Educación (Salazar, 2014), en el 2016 se realiza una reforma que se hace operativa con un diseño curricular renovado, textos guía y capacitaciones para el docente, pero esta articulación entre los tres elementos antes descritos, aunque parece consistente, en la práctica tiene algunos desfases.

La actualización curricular para el área de matemática está fundamentada en la perspectiva epistemológica emergente del tema mencionado, denominada pragmático-constructivista, este modelo considera que el estudiante alcanza aprendizajes significativos cuando resuelve problemas de la vida real, aplicando los conceptos y herramientas matemáticas que aprendió (Ministerio de Educación, 2016a), se aplica un currículo que flexibiliza los temas a desarrollarse (Ministerio de Educación, 2016b) especialmente en matemática; sin embargo, los resultados obtenidos en evaluaciones de matemática continúan siendo consistentemente bajas (Heredia, 2018; Ponce, 2000), con aprendizajes superficiales y memorísticos (Vielma, 2019), donde el docente tiene deficiencias en el abordaje de ciertos temas (Hidalgo, Oquendo, Hidalgo, \& Hidalgo, 2018). Pero estos resultados no son una casualidad, la clase aún es tradicional por diversas razones: el profesor que no tiene formación pedagógica y se siente cómodo con desarrollar la clase de siempre, el docente está forzado a cumplir con los programas hasta el fin del año escolar, por su parte los estudiantes esperan que el docente les enseñe (Pochulu \& Font, 2011) y memorizan los temas para rendir sus evaluaciones (Ponce, 2000).

El docente, por su parte, en un intento por actualizarse da una clase "magistral interactiva", donde todavía goza del prestigio dado por su saber, sin embargo, propicia una interacción que en la práctica es unidireccional con sus discentes, donde él solo aceptará lo que coincida con su propio discurso (Pochulu \& Font, 2011), el docente tiene una preparación libresca y reproductiva (Ortiz, Fabara, Villagomez, \& Hidalgo, 2017), durante mucho tiempo ha dado la misma asignatura y ha tenido poca actualización, así que, repite una y otra vez la misma clase, memorizada de un antiguo texto que en el pasado le sirvió como referente (Bravo, Trelles, \& Barrazueta, 2017).

El gobierno ha tenido entre sus políticas educativas la producción de textos escolares (Ponce, 2000) que vuelven operativa la actualización curricular y ayudan a organizar el desarrollo de los cursos. Actualmente se dispone de textos guía tanto para el estudiante como para el docente, con el fin de apoyar el proceso de enseñanza y aprendizaje. Estos textos tienen afinidad con la propuesta curricular, por lo que desarrollan los temas con actividades contextuales, reflexivas, de trabajo activo, participativo y colaborativo, de uso de materiales concretos y de recursos tecnológicos (Ministerio de Educación, 2016f).

Es claro que en el diseño de la actualización curricular la intención fue elaborar una propuesta constructivista que articule los todos los elementos, entre ellos: currículo, texto y docente, sin embargo al llevar esta propuesta a la práctica, se encuentra el problema: los tres elementos descritos tienen dificultades, ya que la actualización y el texto contienen excesivos temas que toman mucho tiempo desarrollarlos en un paradigma que requiere de reflexión, antes que la 
memorización. El docente aún desarrolla su clase con metodologías tradicionales y tiene recelo de usar las tecnologías, que no compatibiliza con la propuesta del Ministerio de Educación, estas inconsistencias entre los elementos nos llevan más adelante a analizar en detalle esta situación.

\section{Metodología}

En esta investigación se aplica la metodología de revisión documental, mediante una búsqueda sistemática de documentos, se hace un análisis bibliográfico a diferentes textos del Ministerio de Educación que dan soporte al ajuste curricular del 2016, y de otros autores que investigan sobre el tema. El objetivo es analizar relación entre el currículo, texto y docente tal como lo plantean los documentos oficiales del Ministerio.

Posteriormente se analiza lo que sucede en la práctica, que la interrelación entre estos tres elementos no siempre se desarrolla como se espera, y es evidente que se dan desfases que finalmente afectan a la razón de ser del proceso educativo: el estudiante y sus aprendizajes de matemática.

\section{La relación ideal entre el currículo, el texto y el docente de matemática}

Para iniciar el análisis de la relación entre los elementos: diseño curricular, texto guía y docente, revisamos los conceptos fundamentales. El Ministerio de Educación (2016b) menciona que el currículo es la expresión del proyecto educativo del país enfocado al aprendizaje y el desarrollo de todas las personas en el aspecto individual y social. Sobre las guías didácticas, se menciona que desarrollan el currículo y apoyan al docente para "enriquecer los procesos de enseñanza y aprendizaje a partir del contenido del texto de los estudiantes, permitiendo desarrollar los procesos de investigación y de aprendizaje más allá del aula" (Ministerio de Educación, 2016g, p.4). Dado que el currículo está fundamentado en el constructivismo, en el Ecuador, el docente tiene un rol de guía de los estudiantes, que los oriente al logro de competencias al fomentar metodologías activas y participativas que incentiven "el pensamiento racional y crítico, el trabajo individual y cooperativo del alumnado en el aula, que conlleve la lectura y la investigación, así como las diferentes posibilidades de expresión" (Ministerio de Educación, 2016h, p.17).

Con la actualización curricular del 2016 se desarrolla una propuesta más abierta y flexible que responde a los intereses y necesidades de los estudiantes, da atención a la diversidad de los estudiantes, y se adapta a sus diferentes ritmos de aprendizaje (Ministerio de Educación, 2016b). El diseño curricular pretende que se alcancen capacidades expresadas en destrezas con criterios de desempeño, también da al docente orientaciones metodológicas y criterios e indicadores de evaluación (Delgado, Vera, Cruz, \& Pico, 2018); sin embargo, en cada ocasión que se han realizado ajustes curriculares se ha tenido una lógica de incorporación de nuevos contenidos, teniéndose como resultado una sobrecarga de temas a ser desarrollados y aprendidos por los estudiantes, por eso la actualización curricular 2016 distingue entre "aprendizajes imprescindibles" y "aprendizajes deseables" que le da a la institución educativa cierta flexibilidad y le permite establecer prioridades en cuanto a decidir qué temas deseables se desarrollan (Ministerio de Educación, 2016b), que se ajuste a la realidad de la institución y a las necesidades de sus 
estudiantes (Cóndor, Rodríguez, Remache, \& Sánchez, 2018).

Los textos elaborados por el Ministerio de Educación ofrecen al docente una guía, donde se encuentra la planificación de los diferentes bloques curriculares, con las destrezas con criterio de desempeño que se pretenden lograr, una serie de orientaciones metodológicas para la organizar y desarrollar su clase, indicadores de logro y actividades de evaluación (Ministerio de Educación, 2016c). Por su parte, los estudiantes también tienen su texto, donde se desarrolla el currículo de forma integral, adaptado a su edad, y que propicia su desarrollo personal pleno y su integración a la sociedad guiada por los principios del Buen Vivir (Ministerio de Educación, 2016d).

El texto oficial que guía al docente, le apoya a desarrollar la clase de matemática de forma creativa, dándole información contextual e histórica, ampliando conceptos, desarrollando ejercicios modelo, facilitándole metodologías para la resolución de problemas (Ministerio de Educación, 2016e), proponiendo el desarrollo de actividades de investigación, organizando actividades colaborativas, mostrando propuestas para evaluar los aprendizajes, todo esto en concordancia con la perspectiva pragmática constructivista (Ministerio de Educación, 2016a). La actualización curricular incorpora nuevos temas que van cobrando vigencia en el contexto social, por lo tanto, los textos se vuelven bastante abarcadores, y llegan a tener un sinnúmero de temas.

Los textos oficiales de matemática para el estudiante, fomentan el autoaprendizaje, la investigación, el uso de tecnologías de la información y comunicación, ejercicios contextualizados, aplicaciones a la vida real, nos cuenta la historia de la matemática, el propósito es que el estudiante desarrolle confianza en su propio potencial y tenga gusto por la matemática (Ministerio de Educación, 2016f) se espera que el estudiante esté motivado para tener una participación activa en la clase, que gestione el logro de sus aprendizajes, y que estos sean significativos.

Por su parte los docentes que ejercen la docencia en matemática han ganado concursos de oposición y méritos, donde debían demostrar ser competentes en lo disciplinar y lo pedagógico. En el aspecto disciplinar, deben tener una sólida base de conocimientos que les permita desarrollar sus clases con solvencia, que conozcan el contexto histórico del tema, que entiendan a profundidad el tema, que sepan dónde o cómo se aplican esos conocimientos (Bravo, Trelles, \& Barrazueta, 2017). Mientras, en lo pedagógico, los docentes deben planificar y organizar sus clases apegados al paradigma que fundamenta el diseño curricular, deben motivar al estudiante y organizar la clase para que sea él quién pase a tener el rol protagónico de aprender.

La labor del docente es de suma importancia, él debe planificar cuidadosamente una serie de actividades que le propongan al estudiante ser activo, investigador, cooperativo, reflexivo, debe planificar diversas técnicas de evaluación que le ayudarán a optimizar los procesos de aprendizaje de sus estudiantes (Cóndor, Rodríguez, Remache, \& Sánchez, 2018). El Ministerio de Educación, le facilita al docente el texto que guiará las actividades en el aula y que han sido diseñadas en base al paradigma vigente. Para mantener actualizado al docente, también se le ofrece cursos de capacitación de modo que sean capaces de plasmar en sus clases la visión del constructivismo y se logre en los estudiantes aprendizajes significativos y duraderos.

Como consecuencia de ese nivel de integración de los tres elementos del dispositivo educativo: 
diseño curricular, textos y docentes de matemática, se espera que los estudiantes estén motivados por aprender, sean capaces de autorregular sus procesos de aprendizaje, logren aprendizajes mediante construcción reflexiva de conceptos, en fin, los estudiantes deben alcanzar aprendizajes significativos, deben tener competencias que les sirva para aplicar los aprendizajes en la vida cotidiana y en la práctica profesional (Hidalgo, et. al, 2018).

\section{La realidad de la relación entre el currículo, el texto y el docente de matemática}

Existe una buena coherencia entre el diseño curricular del 2016, fundamentado en la perspectiva emergente de la matemática: pragmática-constructivista, y los textos elaborados por el Ministerio de Educación que lo hacen operativo, planteando que el estudiante logre aprendizajes significativos al resolver problemas del contexto y aplicaciones de la vida real (Ministerio de Educación, 2016b), mediante una serie de actividades centradas en la motivación, actividad y reflexión del estudiante.

Pero en la práctica el diseño curricular considera una cantidad excesiva de aprendizajes a ser logrados por el estudiante (Herrera \& Cochancela, 2020), el documento de ajuste curricular categoriza a estos aprendizajes como básicos imprescindibles y básicos deseables (Ministerio de Educación, 2016a), y aunque los primeros son obligatorios, a los segundos da a las instituciones educativas la posibilidad de decidir cuales temas deseables desarrollar. El problema es que las instituciones educativas y los docentes, se presionan por desarrollar todos los aprendizajes del currículo, porque posteriormente en las evaluaciones Ser Bachiller, que sirven como un componente de la nota de graduación y para el ingreso a la universidad, forman parte de la evaluación todos los temas del currículo.

En el caso de los textos, el problema es que traen cantidades excesivas de temas, que le dificultan al docente trabajar y lograr en los estudiantes las destrezas planificadas, más bien el docente se ve apremiado por el tiempo y tan solo logra desarrollar muchos contenidos sin que se logre aprendizajes de calidad en los estudiantes (Ministerio de Educación, 2016a). El texto plantea una serie de actividades basadas en el constructivismo, que requieren de más tiempo que el programado para desarrollarlas adecuadamente y lograr los aprendizajes en los estudiantes. Para complicar el tema, los docentes se han visto abocados a cumplir con una serie de informes y llenado de formularios que les ocupa demasiado tiempo en labores administrativas, dejando escaso tiempo para planificar y preparar adecuadamente la clase.

También ha existido la preocupación por que los docentes se actualicen en los cambios y mejoras que trae el ajuste curricular y puedan llevarla adelante, que esos conceptos filosóficos en los que se fundamenta la reforma curricular puedan ser transformados en buenas prácticas dentro del aula. Sin embargo, los cursos que ofrece el Ministerio de Educación para actualizar a los docentes son escasos y tienen mucha demanda (Ortiz et al., 2017), estos cursos se ofrecen prioritariamente a los docentes del sector fiscal y poco llega a los docentes de instituciones particulares; en definitiva, el proceso de apropiación del ajuste curricular aún no termina (Herrera \& Cochancela, 2020). 
En muchos casos los docentes toman los cursos para cumplir ciertos requisitos que les permitirá ascender de categoría y no realmente para aplicarlos en el aula; en otros casos los capacitadores dan un discurso que explica los fundamentos teóricos del ajuste curricular, con un lenguaje de alto nivel que no llega al docente, de modo que la capacitación no se traduce en metodologías y recursos que permitirían desarrollar una clase basada en el constructivismo, así que no siempre esa capacitación se transforma en una clase constructivista, que es lo que pretende la reforma curricular.

Los docentes de matemática, con frecuencia tienen un título universitario de una profesión técnica, pero no tienen preparación pedagógica, entonces la clase tiene carencias en la parte didáctica y metodológica (Bravo, Trelles, \& Barrazueta, 2017) muchos docentes aún tienen prácticas tradicionales, donde ellos todavía tienen un rol protagónico, deciden qué y cómo hacer las cosas en el aula (Ortiz et al., 2017). Escogen los temas que se desarrollarán en función de lo que conocen desde antaño, y no desarrollan nuevas temáticas que se requieren en la actualidad, en consecuencia, ciertos temas son minimizados o excluidos, otros tiene dificultades con usar en el aula las tecnologías de la información y la comunicación. Aunque, aparentemente lo programado se cumple, pero en la práctica una cosa es el currículo declarado para cumplir con las formalidades académicas, y otro el vivido (Ortiz et al., 2017).

Dado que para el gobierno es una prioridad la inclusión de los sectores tradicionalmente desfavorecidos o con necesidades especiales (Consejo Nacional de Planificación, 2017) Art. 6 de la Ley Orgánica de Educación Intercultural (LOEI, 2010), el docente debe atender a la heterogeneidad de los estudiantes y sus particularidades al enseñar, lo que le obliga al docente a realizar adaptaciones curriculares para el efecto. Todas estas consideraciones hacen que en la práctica el docente no alcanza a desarrollar lo planificado debido a que le toma mucho tiempo.

En el tercer año de bachillerato muchas instituciones educativas deciden preparar a sus estudiantes para la evaluación Ser Bachiller y destinan las últimas semanas del año escolar a este fin, dejándose de lado algunos temas planeados en el currículo. Los estudiantes son los afectados de que en la práctica los tres elementos del dispositivo: diseño curricular, textos y docentes de matemática no engranen adecuadamente y existan los desfases antes mencionados.

Otro desfase se da entre el diseño curricular y el docente de matemática, cuando el currículo de matemática se fundamenta en la perspectiva pragmático-constructivista, donde el estudiante alcanza aprendizajes significativos al resolver problemas de la vida real, en cambio, el docente da una clase que no es constructivista y está basada en el desarrollo teórico, la resolución de ejercicios numéricos y literales que no tienen un contexto ni una aplicación a la vida real, y el estudiante tiene un papel pasivo en la clase, en consecuencia, el estudiante no logra aprendizajes profundos y significativos. En la relación entre el docente y el texto de matemática, también hay dificultades, cuando el docente decide desarrollar temas que son de su dominio y prefiere dejar de lado aquellos que no son su fortaleza, en ese caso el estudiante puede verse privado de ciertos aprendizajes importantes para su formación integral, la relación que se describe entre los tres elementos lo podemos apreciar en la Figura 1. 


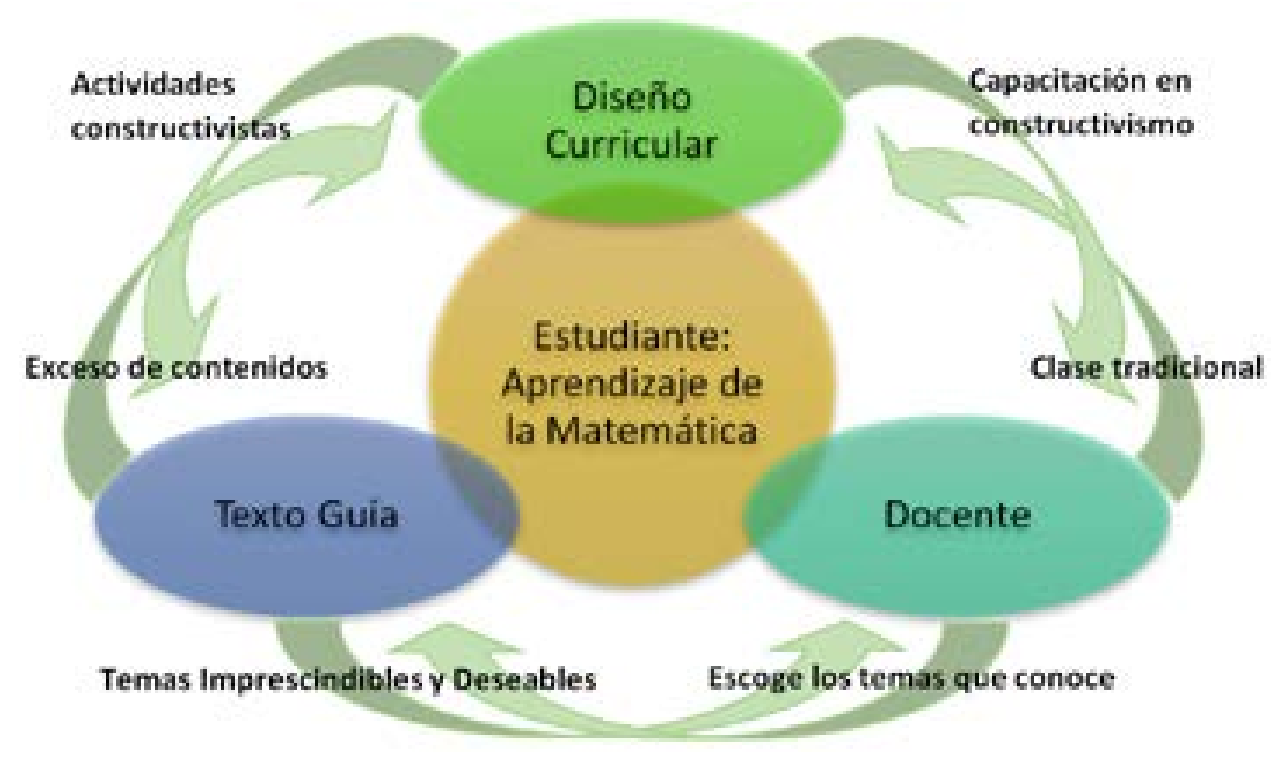

Figura 1. Desfases entre los tres elementos del dispositivo educativo en la clase de Matemática

\section{Conclusiones}

En el papel, los tres elementos del dispositivo educativo: currículo, texto y docente, funcionan según la actualización curricular del 2016, dado que el currículo ha sido diseñado fundamentado en el constructivismo, el texto guía desarrolla metodologías activas, participativas, reflexivas; allí, se fomenta el uso de recursos didácticos que permiten a los estudiantes construir conocimientos, y se prevé un docente capacitado y comprometido con el paradigma, de modo que parece que hay armonía entre los elementos descritos.

Sin embargo, en la práctica se encuentran algunas razones por las que estos tres elementos tienen problemas. El diseño curricular da la posibilidad a las instituciones de decidir cuáles son los aprendizajes imprescindibles y cuáles son los deseables, sin embargo, los exámenes de graduación que también sirven para el ingreso a la universidad, exigen a los estudiantes haber aprendido todos los contenidos. Los textos contienen todos los temas, y los docentes en su afán de cubrir la mayor cantidad de ellos, tratan de pasar muy rápido por todos, sin lograr en sus estudiantes aprendizajes de calidad.

A pesar de que el diseño curricular está fundado en el constructivismo, y el texto también está desarrollado en ese paradigma, el docente aún tiene arraigadas las prácticas tradicionales, con métodos que favorecen la memorización y repetición de los temas, dado que el tiempo es escaso, el docente tiene que "dar clases", dejando poco espacio para desarrollar una clase activa, participativa y reflexiva donde el estudiante tenga un papel preponderante. Aunque el texto recomienda al docente usar algunos recursos didácticos, especialmente tecnológicos, el docente poco conoce del potencial de las TICs, y ese desconocimiento limita la calidad de su clase, y la posibilidad de que sus estudiantes logren aprendizajes de calidad. 
Como resultado de las dificultades entre los tres elementos analizados: currículo, texto y docente, quienes finalmente salen afectados de todas las inconsistencias que se dan en la práctica son los estudiantes. Los diseños curriculares y los textos de matemática deben ser revisados para que no sean excesivos los temas que deben desarrollarse, permitan al docente tratarlos con detalle en una clase activa, reflexiva y contextualizada. El docente debe ser capacitado, de modo que conozca el constructivismo y las herramientas que le permitan llevar ese paradigma a la clase, debe usar las tecnologías como un recurso que le facilita el logro de los aprendizajes de sus estudiantes, de modo que dejen de ser considerados como actores pasivos de su proceso educativo, se sientan motivados, y sean proactivos para lograr aprendizajes significativos y gusto por la matemática.

Existen otros elementos que también pueden considerarse en el hecho educativo y merecen ser investigados: las autoridades de las instituciones educativas, la infraestructura y equipamiento, y el papel de los padres de familia, entre otros, son trascendentales en la educación de los jóvenes. 


\section{Referencias bibliográficas}

Bravo, F., Trelles, C., \& Barrazueta, J. F. (2017). Reflexiones sobre la evolución de la clase de matemáticas en el bachillerato ecuatoriano. INNOVA Research Journal, 2(7), 1-12.

Cóndor, B., Rodríguez, Y., Remache, M., \& Sánchez, M. (2019). ¿Cómo llevar el currículo al aula?. Revista Científica Retos de la Ciencia, 3(1), 84-103.

Delgado, J., Vera, M., Cruz, J., \& Pico, J. (2018). El currículo de la educación básica ecuatoriana: Una mirada desde la actualidad. Revista Cognosis, 3(4), 47-66.

Herrera, M., \& Cochancela, M. (2020). Aportes de las reformas curriculares a la educación obligatoria en el Ecuador. Revista Scientific, 5(15), 362-383

Ministerio de Educación. (2010). Ley Orgánica de Educación Intercultural. Ecuador: Mineduc.

Ministerio de Educación. (2016a). Currículo de los niveles de educación obligatoria. Ecuador: Mineduc.

Ministerio de Educación. (2016b). Introducción Matemática. Ecuador: Mineduc.

Ministerio de Educación. (2016c). Matemática 10 Grado Guía del Docente. Ecuador: Mineduc.

Ministerio de Educación. (2016d). Matemática $1^{\circ}$ Curso Texto del Estudiante. Ecuador: Mineduc.

Ministerio de Educación. (2016e). Precisiones Curriculares para el Bachillerato General Unificado. Ecuador: Mineduc.

Ministerio de Educación. (2016f). Lineamientos Curriculares para el Bachillerato General Unificado. Ecuador: Mineduc.

Ministerio de Educación. (2016g). Matemática $1{ }^{\circ}$ Curso Guía del Docente. Ecuador: Mineduc.

Ministerio de Educación. (2016h). Introducción General. Ecuador: Mineduc.

Consejo Nacional de Planificación. (2017). Plan Nacional de Desarrollo 2017-2021-Toda una Vida. Ecuador.

Heredia, V. $(2018,12,12)$. Resultados de la evaluación PISA-D plantean varios retos en educación. El Comercio. Recuperado de: https://www.elcomercio.com/ actualidad/resultados-evaluacion-pisa-retos-educacion. html

Hidalgo, D., Oquendo, V., Hidalgo, B., \& Hidalgo, I. (2018). Competencias que poseen los bachilleres en el ámbito de la matemática. Revista CienciAmérica, 7(2), 57-70. 
Ortiz, M.E., Fabara, E., Villagómez, M.S. \& Hidalgo, L. (2017). La Formación y el Trabajo Docente en el Ecuador. Abya Yala. Cuenca, Ecuador.

Pochulu, M., \& Font, V. (2011). Análisis del funcionamiento de una clase de matemáticas no significativa. Revista latinoamericana de investigación en matemática educativa, 14(3), 361-394.

Ponce, J. (2000). La educación básica en el Ecuador: Problemas y propuestas de solución. SIISE. Quito, Ecuador.

Salazar, R. (2014). Sistema educativo ecuatoriano: Una revisión histórica hasta nuestros días. Guayaquil, Ecuador.

Vielma, J. (2019, 01, 26). Ecuador reprobó en matemáticas a nivel internacional. El Universo. Recuperado de: https://www.eluniverso.com/guayaquil/2019/02/26/ nota /7207946/matematicas-no-se-paso-prueba 


\section{Copyright (c) 2020 Fabián Eugenio Bravo Guerrero}

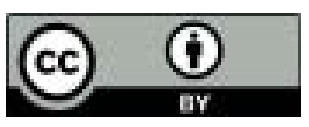

Este texto está protegido bajo una licencia internacional Creative Commons 4.0.

Usted es libre para Compartir-copiar y redistribuir el material en cualquier medio o formato - y Adaptar el documento - remezclar, transformar y crear a partir del material-para cualquier propósito, incluso para fines comerciales, siempre que cumpla las condiciones de Atribución. Usted debe dar crédito a la obra original de manera adecuada, proporcionar un enlace a la licencia, e indicar si se han realizado cambios. Puede hacerlo en cualquier forma razonable, pero no de forma tal que sugiera que tiene el apoyo del licenciante o lo recibe por el uso que hace de la obra.

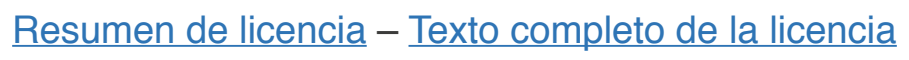

